\title{
A criterion for virtual global generation
}

\author{
INDRANIL BISWAS AND A. J. PARAMESWARAN
}

\begin{abstract}
Let $X$ be a smooth projective curve defined over an algebraically closed field $k$, and let $F_{X}$ denote the absolute Frobenius morphism of $X$ when the characteristic of $k$ is positive. A vector bundle over $X$ is called virtually globally generated if its pull back, by some finite morphism to $X$ from some smooth projective curve, is generated by its global sections. We prove the following. If the characteristic of $k$ is positive, a vector bundle $E$ over $X$ is virtually globally generated if and only if $\left(F_{X}^{m}\right)^{*} E \cong E_{a} \oplus E_{f}$ for some $m$, where $E_{a}$ is some ample vector bundle and $E_{f}$ is some finite vector bundle over $X$ (either of $E_{a}$ and $E_{f}$ are allowed to be zero). If the characteristic of $k$ is zero, a vector bundle $E$ over $X$ is virtually globally generated if and only if $E$ is a direct sum of an ample vector bundle and a finite vector bundle over $X$ (either of them are allowed to be zero).
\end{abstract}

Mathematics Subject Classification (2000):14H60 (primary);14F05 (secondary).

\section{Introduction}

Fix an algebraically closed field $k$; the characteristic of $k$ will be denoted by $p$. Let $X$ be an irreducible smooth projective curve defined over $k$.

A vector bundle $E$ over $X$ is said to be virtually globally generated if there exists a smooth projective curve $Y$ defined over $k$ and a surjective morphism

$$
f: Y \longrightarrow X
$$

such that the vector bundle $f^{*} E$ is generated by its global sections. Our aim here is to give a necessary and sufficient condition for a vector bundle over $X$ to be virtually globally generated. Before we state the condition, we need to recall the definition of a finite vector bundle.

A vector bundle $E$ over $X$ is called finite if there is a finite étale Galois covering

$$
\gamma: X^{\prime} \longrightarrow X
$$

such that $\gamma^{*} E$ is trivializable; see [No]. Any finite vector bundle is of degree zero. If $k=\mathbb{C}$, then a vector bundle is finite if and only if it admits a flat holomorphic connection whose monodromy is a finite group.

Received June 22, 2005; accepted in revised form January 17, 2006. 
If the characteristic $p>0$, then $F_{X}$ will denote the absolute Frobenius morphism of $X$.

We prove the following theorem (Theorem 4.1):

Theorem 1.1. Assume that $p>0$. A vector bundle $E$ over $X$ is virtually globally generated if and only if there is a positive integer $m$ such that

$$
\left(F_{X}^{m}\right)^{*} E \cong E_{a} \oplus E_{f}
$$

where $E_{a}$ is some ample vector bundle and $E_{f}$ is some finite vector bundle over $X$ (any of $E_{a}$ and $E_{f}$ are allowed to be zero).

Assume that $p=0$. A vector bundle $E$ over $X$ is virtually globally generated if and only if

$$
E \cong E_{a} \oplus E_{f},
$$

where $E_{a}$ is some ample vector bundle and $E_{f}$ is some finite vector bundle over $X$ (any of $E_{a}$ and $E_{t}$ are allowed to be zero).

As a corollary, any ample vector bundle over a smooth projective curve is virtually globally generated.

The unpublished manuscript $[\mathrm{Pa}]$ contained some of the results proved here.

\section{Degree of the universal subbundle}

In this section we assume that the characteristic of the field $k$ to be zero. Fix an irreducible smooth projective curve $X$ of genus $g$ defined over the field $k$.

For a vector bundle $V$ over $X$, the projective bundle over $X$ parametrizing all one dimensional quotients of the fibers of $V$ will be denoted by $\mathbb{P}(V)$. The tautological line bundle over $\mathbb{P}(V)$ will be denoted by $\mathcal{O}_{\mathbb{P}(V)}(1)$. We recall that $V$ is called ample if the line bundle $\mathcal{O}_{\mathbb{P}(V)}(1)$ is so. The slope $\mu(V)$ of $V$ is defined as $\mu(V):=\frac{\operatorname{degree}(V)}{\operatorname{rank}(V)}$. A vector bundle $V$ over $X$ is called semistable if $\mu(W) \leq \mu(V)$ for every subbundle $0 \neq W \subset V$.

Given a vector bundle $V$ over $X$, there is a unique filtration of subbundles

$$
0=V_{0} \subset V_{1} \subset \cdots \subset V_{\ell-1} \subset V_{\ell}=V,
$$

known as the Harder-Narasimhan filtration, which has the following properties: Each successive quotient $V_{i} / V_{i-1}, i \in[1, \ell]$, is semistable, and $\mu\left(V_{i} / V_{i-1}\right)>$ $\mu\left(V_{i+1} / V_{i}\right)$ for all $i \in[1, \ell-1]$. The vector bundle $V_{1}$ (respectively, $V_{\ell} / V_{\ell-1}$ ) is denoted by $V_{\max }$ (respectively, $\left.V_{\min }\right)$, and the rational number $\mu\left(V_{\max }\right)$ (respectively, $\left.\mu\left(V_{\min }\right)\right)$ is denoted by $\mu_{\max }(V)$ (respectively, $\mu_{\min }(V)$ ). The following lemma is well-known.

Lemma 2.1. For any vector bundles $V, W$, and $W^{\prime}$ over $X$ we have:

(a) $\mu_{\max }(V \otimes W)=\mu_{\max }(V)+\mu_{\max }(W)$. 
(b) $\mu_{\max }\left(S^{n} V\right)=n \cdot \mu_{\max }(V)$, where $S^{n} V$ is the $n$-fold symmetric power of $V$.

(c) $\mu_{\min }\left(V^{*}\right)=-\mu_{\max }(V)$ and $\mu_{\max }\left(V^{*}\right)=-\mu_{\min }(V)$, where $V^{*}$ is the dual of $V$.

(d) If $\mu_{\max }(V)<0$, then $H^{0}(X, V)=0$.

(e) If $V \longrightarrow W$ is a surjective homomorphism with $V$ ample, then $W$ is ample.

(f) If $0 \longrightarrow W^{\prime} \longrightarrow V \longrightarrow W \longrightarrow 0$ is a short exact sequence of vector bundles with $W$ and $W^{\prime}$ ample, then $V$ is ample.

(g) A vector bundle $V$ over $X$ is ample if and only if $\mu_{\min }(V)>0$.

(h) Any pull back of a virtually globally generated vector bundle is again virtually globally generated.

(i) If $V$ is semistable (respectively, ample), and $f: D \longrightarrow X$ is a finite morphism from a projective curve $D$, then $f^{*} V$ is semistable (respectively, ample).

The statements (a) and (b) in Lemma 2.1 follow from the fact that the tensor product of two semistable vector bundles is again semistable, provided the characteristic of the base field is zero (see [RR, Theorem 3.18]). Statements (c) and (d) are obvious. For the rest, see [Ha1] and [Ha2, Proposition 2.1(ii)]. That the pull back of a semistable vector bundle is semistable follows from the fact that the HarderNarasimhan filtration for a pull back by a finite map descends, and the descended filtration coincides with the Harder-Narasimhan filtration of the original bundle.

Remark 2.2. When the characteristic of the base field is positive, the tensor product of two semistable vector bundles need not be semistable. For this reason we have to treat separately the two cases of zero characteristic and positive characteristic.

For a vector bundle $V$ over $X$ and a divisor $D$ on $X$, the vector bundle $V \bigotimes_{\mathcal{O}_{X}} \mathcal{O}_{X}(D)$ will be denoted by $V(D)$.

Lemma 2.3. Take a vector bundle $V$ over $X$. Let $L$ be a line bundle over $X$ and $n$ a positive integer such that degree $(L)>2 g-n \cdot \mu_{\min }(V)$ (as before, $g=\operatorname{genus}(X)$ ). Then the line bundle $\mathcal{O}_{\mathbb{P}(V)}(n) \otimes \pi^{*} L$ over $\mathbb{P}(V)$ is very ample, where $\pi$ is the natural projection of $\mathbb{P}(V)$ to $X$.

Proof. For any two points $x, y \in X$, by Serre duality,

$$
H^{1}\left(X, S^{n} V \otimes L(-x-y)\right)=H^{0}\left(X, S^{n} V^{*} \otimes L^{-1}(x+y) \otimes K_{X}\right)^{*} .
$$

Therefore, in view of Lemma 2.1(d), to prove that

$$
H^{1}\left(X, S^{n} V \otimes L(-x-y)\right)=0
$$

it suffices to show that $\mu_{\max }\left(S^{n} V^{*} \otimes L^{-1}(x+y) \otimes K_{X}\right)<0$. Using Lemma 2.1(a), (b) and (c) we have:

$$
\mu_{\max }\left(S^{n} V^{*} \otimes L^{-1}(x+y) \otimes K_{X}\right)=-n \mu_{\min }(V)-\operatorname{degree}(L)+2 g,
$$

which is negative if and only if degree $(L)>2 g-n \mu_{\min }(V)$. 
Since (2.2) holds, for any two distinct points $x, y \in X$, the evaluation map

$$
H^{0}\left(X, S^{n} V \otimes L\right) \longrightarrow\left(S^{n} V \otimes L\right)_{x} \oplus\left(S^{n} V \otimes L\right)_{y}
$$

is surjective. This implies that the global sections of $\mathcal{O}_{\mathbb{P}(V)}(n) \otimes \pi^{*} L$ separate points of $\mathbb{P}(V)$, where $\pi$ is the projection of $\mathbb{P}(V)$ to $X$.

To prove that the sections of $\mathcal{O}_{\mathbb{P}(V)}(n) \otimes \pi^{*} L$ also separate tangent vectors of $\mathbb{P}(V)$ we proceed as follows. Take any point $z \in \mathbb{P}(V)$, and take any nonzero tangent vector $v \in T_{z} \mathbb{P}(V)$. Set $x:=\pi(z)$. If $v$ is tangent to the fiber $\pi^{-1}(x)=$ $\mathbb{P}\left(V_{x}\right)$, then there is a section

$$
\sigma \in H^{0}\left(\mathbb{P}\left(V_{x}\right), \mathcal{O}_{\mathbb{P}\left(V_{x}\right)}(n)\right)
$$

such that $\sigma(z)=0$ and $v$ is transversal to the divisor of $\sigma$. Let

$$
\widehat{\sigma} \in H^{0}\left(\mathbb{P}(V), \mathcal{O}_{\mathbb{P}(V)}(n) \otimes \pi^{*} L\right)
$$

be a lift of the section $\sigma$; since the homomorphism in (2.3) is surjective, such a lift $\widehat{\sigma}$ exists. The section $\widehat{\sigma}$ clearly separates $v$.

Now assume that $v \in T_{Z} \mathbb{P}(V)$ is not tangent to $\mathbb{P}\left(V_{x}\right)$. Consider the long exact sequence of cohomologies for the short exact sequence of sheaves

$$
0 \longrightarrow S^{n} V \otimes L(-2 x) \longrightarrow S^{n} V \otimes L(-x) \longrightarrow\left(S^{n} V \otimes L(-x)\right)_{x} \longrightarrow 0 .
$$

Since $H^{1}\left(X, S^{n} V \otimes L(-2 x)\right)=0$ (see (2.2)), from this long exact sequence we conclude that the evaluation map

$$
H^{0}\left(X, S^{n} V \otimes L(-x)\right) \longrightarrow\left(S^{n} V \otimes L(-x)\right)_{x}
$$

is surjective. Consequently, there is a section

$$
\sigma \in H^{0}\left(\mathbb{P}(V), \mathcal{O}_{\mathbb{P}(V)}(n) \otimes \pi^{*} L(-x)\right)
$$

such that $\sigma(z) \neq 0$. Therefore, $z$ is a smooth point of the divisor on $\mathbb{P}(V)$ for the section of $\mathcal{O}_{\mathbb{P}(V)}(n) \otimes \pi^{*} L$ given by $\sigma$, and furthermore, the divisor is transversal to $v$. This completes the proof of the lemma.

Given a vector bundle $V$ over $X$, consider the canonical universal exact sequence of vector bundles on $\mathbb{P}(V)$ :

$$
0 \longrightarrow \mathcal{K}(V) \longrightarrow \pi^{*} V \longrightarrow \mathcal{O}_{\mathbb{P}(V)}(1) \longrightarrow 0,
$$

where $\pi$ as before is the projection of $\mathbb{P}(V)$ to $X$.

Lemma 2.4. Let $V, L$ and $n$ be as in Lemma 2.3. Let $D$ be an irreducible complete intersection curve in $\mathbb{P}(V)$ obtained by intersecting divisors on $\mathbb{P}(V)$ lying in the complete linear system for the very ample line bundle $\mathcal{O}_{\mathbb{P}(V)}(n) \otimes \pi^{*} L$. Then

$$
\operatorname{degree}\left(\left.\mathcal{K}(V)\right|_{D}\right)=-(r-1) n^{r-2} \operatorname{degree}(L),
$$

where $r=\operatorname{rank}(V)$. 
Proof. Since $D$ is a complete intersection curve, degree $\left(\left.\mathcal{O}_{\mathbb{P}(V)}(1)\right|_{D}\right) \in \mathbb{Z}$ coincides with the cup product

$$
c_{1}\left(\mathcal{O}_{\mathbb{P}(V)}(1)\right) \cup c_{1}\left(\mathcal{O}_{\mathbb{P}(V)}(n) \otimes \pi^{*} L\right)^{r-1} \in H^{2 r}(\mathbb{P}(V), \mathbb{Z})=\mathbb{Z} .
$$

For any line bundle $\xi$ on $\mathbb{P}(V)$, let $[\xi] \in H^{2}(\mathbb{P}(V), \mathbb{Z})$ denote the first Chern class of $\xi$.

Note that $\left[\pi^{*} L\right] \cup\left[\pi^{*} L\right]=0$. Also, for any fiber $F:=\pi^{-1}(x)$ we have

$$
\left[\mathcal{O}_{\mathbb{P}(V)}(1)\right]^{r-1} \cap[F]=1,
$$

where $[F] \in H_{2 r-2}(\mathbb{P}(V), \mathbb{Z})$ is the homology class of the fiber. This implies that $\left[\mathcal{O}_{\mathbb{P}(V)}(1)\right]^{r-1} \cup\left[\pi^{*} L\right]=\operatorname{degree}(L)$. We also have

$$
\left[\mathcal{O}_{\mathbb{P}(V)}(1)\right]^{r}=\operatorname{degree}(V) .
$$

Combining all these we get the following:

$$
\begin{aligned}
& \operatorname{degree}\left(\left.\mathcal{O}_{\mathbb{P}(V)}(1)\right|_{D}\right)=[D] \cup\left[\mathcal{O}_{\mathbb{P}(V)}(1)\right] \\
& =\left(\left[\mathcal{O}_{\mathbb{P}(V)}(n)\right]+\left[\pi^{*} L\right]\right)^{r-1} \cup\left[\mathcal{O}_{\mathbb{P}(V)}(1)\right] \\
& =\left(\left[\mathcal{O}_{\mathbb{P}(V)}(n)\right]^{r-1}+(r-1)\left[\mathcal{O}_{\mathbb{P}(V)}(n)\right]^{r-2} \cup\left[\pi^{*} L\right]\right) \cup\left[\mathcal{O}_{\mathbb{P}(V)}(1)\right] \\
& =n^{r-2}\left[\mathcal{O}_{\mathbb{P}(V)}(1)\right]^{r-1} \cup\left(n\left[\mathcal{O}_{\mathbb{P}(V)}(1)\right]+(r-1)\left[\pi^{*} L\right]\right) \\
& =n^{r-2}(\operatorname{degree}(V) n+\operatorname{degree}(L)(r-1)) .
\end{aligned}
$$

Now, since the degree of the restriction map $\left.\pi\right|_{D}: D \longrightarrow C$ is $n^{r-1}$, the lemma follows from the universal exact sequence (2.4) and the above expression for $\operatorname{degree}\left(\left.\mathcal{O}_{\mathbb{P}(V)}(1)\right|_{D}\right)$.

\section{Ample subbundles of ample vector bundles}

Throughout this section we will continue with the assumption that the characteristic of $k$ is zero.

Proposition 3.1. Let $X$ is an irreducible smooth projective curve of genus $g$. Let $V$ be an ample vector bundle of rank $r$ over $X$, with $r \geq 2$. Assume that either $r=2$ or $V$ is semistable. Then there exists a smooth irreducible curve $D$ in $\mathbb{P}(V)$ as in Lemma 2.4 such that the restriction $\left.\mathcal{K}(V)\right|_{D}$ is ample, where $\mathcal{K}(V)$ is defined in (2.4). 
Proof. Fix an integer $n>\frac{(r-1)(2 g+2)}{\mu_{\min }(V)}$. Then there is an integer $d<0$ such that

$$
-\frac{(r-2) n \mu_{\min }(V)}{r-1}>d>2 g-n \mu_{\min }(V) \text {. }
$$

Let $L$ be a line bundle over $X$ of degree $d$. Then $\mathcal{O}_{\mathbb{P}(V)}(n) \otimes \pi^{*} L$ is very ample by Lemma 2.3 and the inequality (3.1). Take any complete intersection curve $D$ as in Lemma 2.4. As degree $(L)=d<0$, using Lemma 2.4,

$$
\operatorname{degree}\left(\left.\mathcal{K}(V)\right|_{D}\right)=-(r-1) n^{r-2} \operatorname{degree}(L)>0 .
$$

Hence, if $r=2$, then the line bundle $\left.\mathcal{K}(V)\right|_{D}$ is ample.

Assume now that the vector bundle $V$ is semistable. Take any $D$ as in Lemma 2.4. By Lemma 2.1(i), the restriction $\left(\left.\pi\right|_{D}\right)^{*} V=\left.\left(\pi^{*} V\right)\right|_{D}$ is semistable. Then by Lemma 2.4,

$$
\operatorname{degree}\left(\left.\mathcal{K}(V)\right|_{D}\right)=-(r-1) n^{r-2} \operatorname{degree}(L) .
$$

Hence from (3.1) we conclude that

$$
\operatorname{degree}\left(\left.\mathcal{K}(V)\right|_{D}\right)>(r-2) n^{r-1} \mu(V)
$$

$\left(\operatorname{as} \mu_{\min }(V)=\mu(V)\right.$ and $\left.d=\operatorname{degree}(L)\right)$.

To prove that $\left.\mathcal{K}(V)\right|_{D}$ is ample we need to show that $\mu_{\min }\left(\left.\mathcal{K}(V)\right|_{D}\right)>0$ (see Lemma $2.1(\mathrm{~g})$ ). Assume that $\mu_{\min }\left(\left.\mathcal{K}(V)\right|_{D}\right) \leq 0$. Then there is a subbundle $\left.K^{\prime} \subset \mathcal{K}(V)\right|_{D}$ with degree $\left(K^{\prime}\right) \geq \operatorname{degree}\left(\left.\mathcal{K}(V)\right|_{D}\right)$ (take $K^{\prime}$ to be the kernel of the projection $\left.\left.\left.\mathcal{K}(V)\right|_{D} \longrightarrow \mathcal{K}(V)\right|_{D} /\left(\left.\mathcal{K}(V)\right|_{D}\right)_{\text {min }}\right)$. Combining this with (3.2) we have

$$
\begin{aligned}
\mu\left(K^{\prime}\right) & :=\frac{\operatorname{degree}(K)}{\operatorname{rank}(K)} \geq \frac{\operatorname{degree}\left(\left.\mathcal{K}(V)\right|_{D}\right)}{r-2}>\frac{(r-2) n^{r-1} \mu(V)}{r-2} \\
& =n^{r-1} \mu(V)=\mu\left(\left.\pi^{*} V\right|_{D}\right) .
\end{aligned}
$$

The above inequality $\mu\left(K^{\prime}\right)>\mu\left(\left(\left.\pi\right|_{D}\right)^{*} V\right)$ contradicts the semistability condition of $\left(\left.\pi\right|_{D}\right)^{*} V$. Consequently, $\mu_{\min }\left(\left.\mathcal{K}(V)\right|_{D}\right)>0$, which implies that $\left.\mathcal{K}(V)\right|_{D}$ is ample. This completes the proof of the proposition.

Remark 3.2. In the proof of Proposition 3.1 we saw that if $n>\frac{(r-1)(2 g+2)}{\mu_{\min }(V)}$ and $d:=$ degree $(L)$ satisfies (3.1), then $\mathcal{O}_{\mathbb{P}(V)}(n) \otimes \pi^{*} L$ is ample. If, furthermore, $\operatorname{rank}(V)=2$, then we saw that for any complete intersection curve $D$ as in Lemma 2.4 , the restriction of the line bundle $\mathcal{K}(V)$ to $D$ is ample. Therefore, if $\operatorname{rank}(V)=$ 2 , then there are closed curves $D_{1}, D_{2}, D_{3} \subset \mathbb{P}(V)$ such that

$$
\bigcap_{i=1}^{3} D_{i}=\emptyset
$$

and all the restrictions $\left.\mathcal{K}(V)\right|_{D_{i}}, i \in[1,3]$, are ample. 
Corollary 3.3. Let $V$ be an ample vector bundle over $X$ of rank at least two. There is a finite map $f: Y \longrightarrow X$, where $Y$ is a smooth projective curve, such that $f^{*} V$ admits an ample subbundle of co-rank one.

Proof. If $V$ is semistable, then this follows from Proposition 3.1. Assume that $V$ is not semistable. Let

$$
\psi: V \longrightarrow V_{\min }
$$

be the quotient homomorphism; the quotient $V_{\min }$ is defined in Section 2. As $V$ is ample, we have degree $\left(V_{\min }\right)>0$. Therefore, each successive quotient in the Harder-Narasimhan filtration of $V$ is ample (follows from Lemma 2.1(g)). Now using Lemma 2.1(f) we conclude that the subbundle $\operatorname{kernel}(\psi) \subset V$ is ample.

Therefore, if $V_{\min }$ is a line bundle, then $\operatorname{kernel}(\psi)$ is an ample subbundle of $V$ of co-rank one.

Assume now that $\operatorname{rank}\left(V_{\min }\right) \geq 2$. Then from Proposition 3.1 it follows that there exists a finite morphism $f: Y \longrightarrow X$, where $Y$ is a smooth projective curve, such that there is an ample subbundle $V^{\prime} \subset f^{*} V_{\min }$ of co-rank one. The inverse image $\left(f^{*} \psi\right)^{-1}\left(V^{\prime}\right)$, where $f^{*} \psi$ is the pull back of the homomorphism $\psi$ to $Y$, fits in the following short exact sequence of vector bundles over $Y$

$$
0 \longrightarrow f^{*} \operatorname{kernel}(\psi) \longrightarrow\left(f^{*} \psi\right)^{-1}\left(V^{\prime}\right) \longrightarrow V^{\prime} \longrightarrow 0 .
$$

The vector bundle $f^{*} \operatorname{kernel}(\psi)$ is ample as $\operatorname{kernel}(\psi)$ is so (see Lemma 2.1(i)). Therefore, using Lemma 2.1(f), from the above exact sequence it follows that the vector bundle $\left(f^{*} \psi\right)^{-1}\left(V^{\prime}\right)$ is ample. Hence, $\left(f^{*} \psi\right)^{-1}\left(V^{\prime}\right) \subset f^{*} V$ is an ample subbundle of co-rank one.

Proposition 3.4. Let $V$ be an ample vector bundle over $X$ of rank at least two. Then there exists an irreducible smooth projective curve $Y$ and a finite morphism $f: Y \longrightarrow X$ with the following property: there are finitely many ample subbundles of co-rank one

$$
\mathcal{K}_{i} \subset f^{*} V,
$$

$i \in[1, n]$, such that the natural homomorphism

$$
\bigoplus_{i=1}^{n} \mathcal{K}_{i} \longrightarrow f^{*} V
$$

is surjective.

Proof. First assume that $\operatorname{rank}(V)=2$. There are curves $D_{1}, D_{2}, D_{3} \subset \mathbb{P}(V)$ such that the restrictions $\xi_{i}:=\left.\mathcal{K}(V)\right|_{D_{i}}, i \in[1,3]$, are ample line bundles and $\bigcap_{i=1}^{3} D_{i}=\emptyset$; see Remark 3.2. Let

$$
v: Y \longrightarrow D_{1} \times{ }_{X} D_{2} \times{ }_{X} D_{3}
$$

be the normalization of an irreducible component of the fiber product over $X$. 
The projection from $Y$ to $D_{i}$ will be denoted by $v_{i}$; the projection from $Y$ to $X$ will be denoted by $v$. Then $v_{i}^{*} \xi_{i}$ are ample by Lemma 2.1(i), and they together generate $\nu^{*} V$ as $\bigcap_{i=1}^{3} D_{i}=\emptyset$.

Now assume that $\operatorname{rank}(V)>2$. Applying Corollary 3.3 twice we obtain a finite covering $f: D \longrightarrow X$ and an ample subbundle $\mathcal{K}$ of $f^{*} V$ such that $\operatorname{rank}\left(f^{*} V / \mathcal{K}\right)=2$. We note that $f^{*} V$ is ample on $D$ by Lemma 2.1(i), and hence $f^{*} V / \mathcal{K}$ is ample by Lemma $2.1(\mathrm{e})$. Replacing $(X, V)$ by $\left(D, f^{*} V / \mathcal{K}\right)$ in the first part of the proof we conclude that there is a finite covering $f_{0}: Y \longrightarrow D$ and ample line subbundles $L_{i} \subset f_{0}^{*}\left(f^{*} V / \mathcal{K}\right), i=1,2,3$, such that $L_{i}$ together generate $f_{0}^{*}\left(f^{*} V / \mathcal{K}\right)$.

Let

$$
\psi: f_{0}^{*} f^{*} V \longrightarrow f_{0}^{*}\left(f^{*} V / \mathcal{K}\right)
$$

be the quotient map. Then $\psi^{-1}\left(L_{i}\right), i \in[1,3]$, are ample by Lemma 2.1(f). Clearly they together generate $f_{0}^{*} f^{*} V$. This completes the proof of the proposition.

The following lemma is well known; see $[\mathrm{Mu}]$.

Lemma 3.5. Let $Z$ be an irreducible nonsingular projective variety defined over $k$. Let $L$ be a line bundle over $X$ such that the line bundle $L^{m}$ has a nonzero section $\sigma$ for some positive integer $m$. Then there exists a finite cyclic covering $f: Z^{\prime} \longrightarrow Z$ (possibly ramified) such that the line bundle $f^{*} L$ has a nonzero section $\sigma^{\prime}$ satisfying the condition $f\left(\operatorname{Div}\left(\sigma^{\prime}\right)\right)=\operatorname{Div}(\sigma)$. Moreover, if the divisor $\operatorname{Div}(\sigma)$ is smooth, then $Z^{\prime}$ and $f\left(\operatorname{Div}\left(\sigma^{\prime}\right)\right)$ can be chosen to be smooth.

Proof. Let $\pi$ denote the projection to $Z$ of the total space of $L$. Let $Z^{\prime}$ be the subvariety of the total space of $L$ defined by:

$$
Z^{\prime}:=\left\{z \in L \mid z^{m}=\sigma(\pi(z))\right\} .
$$

Let $f: Z^{\prime} \longrightarrow Z$ be the restriction of $\pi$. We have the section $\sigma^{\prime}$ of $f^{*} L$ that sends any $y \in Z^{\prime} \subset L$ to $(y, y) \in f^{*} L \cong L \times{ }_{Z} Z^{\prime}$. The pair $\left(Z^{\prime}, \sigma^{\prime}\right)$ satisfy all the conditions in the lemma.

Theorem 3.6. Let $V$ be an ample vector bundle over an irreducible smooth projective curve $X$ defined over an algebraically closed field $k$ of characteristic zero. Then $V$ is virtually globally generated.

Proof. First assume that $V$ is an ample line bundle. Choose an integer $m \gg 0$ such that $V^{\otimes m}$ is a very ample line bundle. Fix two sections

$$
\tau_{1}, \tau_{2} \in H^{0}\left(X, V^{\otimes m}\right)
$$

having only simple zeros such that $\operatorname{Div}\left(\tau_{1}\right) \bigcap \operatorname{Div}\left(\tau_{2}\right)=\emptyset$. Then by Lemma 3.5 one can have two coverings

$$
f_{i}: Y_{i} \longrightarrow X
$$


and sections $\sigma_{i} \in H^{0}\left(Y_{i}, f_{i}^{*} V\right), i=1,2$, with the property that $\sigma_{i}$ vanishes on $\operatorname{Div}\left(\tau_{i}\right)$. Let $Y$ be the normalization of an irreducible component of the fiber product $Y_{1} \times{ }_{X} Y_{2}$ over $X$. The pull back of the two sections $\sigma_{1}$ and $\sigma_{2}$ to $Y$ together generate the pull back of $V$ to $Y$.

Now we assume that $r:=\operatorname{rank}(V) \geq 2$. We will employ induction on $r$. We assume that any ample vector bundle of rank at most $r-1$ over a smooth projective curve is virtually globally generated.

By Proposition 3.4, there is an irreducible smooth projective curve $D_{0}$ and a finite morphism

$$
f_{0}: D_{0} \longrightarrow X
$$

with the following property: there are ample subbundles co-rank one $\mathcal{K}_{i} \subset f_{0}^{*} V$, $i=1, \cdots, s$, over $D_{0}$ generating $f_{0}^{*} V$. Consequently, by our induction hypothesis, each $\mathcal{K}_{i}, i=1, \cdots, s$, is virtually globally generated. Therefore, there is an irreducible smooth projective curve $Y$ and a projection

$$
h: Y \longrightarrow D_{0},
$$

such that the vector bundle $h^{*} \mathcal{K}_{i}$ over $Y$ is globally generated for each $i \in[1, s]$.

As $\mathcal{K}_{i}, i=1, \cdots, s$, together generate $f_{0}^{*} V$, we conclude that $h^{*} \mathcal{K}_{i}, i \in$ $[1, s]$, together generate $h^{*} f_{0}^{*} V$. Since each $h^{*} \mathcal{K}_{i}$ is globally generated, this implies that $h^{*} f_{0}^{*} V$ is also globally generated. This completes the proof of the theorem.

\section{Criterion for virtual global generation}

Let $k$ be an algebraically closed field of arbitrary characteristic. Let $X$ be an irreducible smooth projective curve defined over $k$. In the introduction we recalled the definition of a finite vector bundle. A vector bundle $V$ over $X$ is called essentially finite if degree $(V)=0$, and there exists a finite vector bundle $V^{\prime}$ and a quotient bundle $Q$ of degree zero of $V^{\prime}$ such that $V$ is isomorphic to a subbundle of $Q$; see [No].

If the characteristic of $k$ is positive, we will denote by $F_{X}$ the absolute Frobenius morphism of $X$. For any integer $m \geq 1$, the $m$-fold iteration of the selfmorphism $F_{X}$ will be denoted by $F_{X}^{m}$. If the characteristic of $k$ is positive, a vector bundle $V$ over $X$ is called strongly semistable if $\left(F_{X}^{m}\right)^{*} V$ is semistable for all $m \geq 1$.

Theorem 4.1. Assume that the characteristic $p=0$. A vector bundle $E$ over $X$ is virtually globally generated if and only if

$$
E \cong E_{a} \oplus E_{f}
$$

where $E_{a}$ is some ample vector bundle and $E_{f}$ is some finite vector bundle over $X$ (any of $E_{a}$ and $E_{t}$ are allowed to be zero). 
Assume that the characteristic $p>0$. A vector bundle $E$ over $X$ is virtually globally generated if and only if there is a positive integer $m$ such that

$$
\left(F_{X}^{m}\right)^{*} E \cong E_{a} \oplus E_{f}
$$

where $E_{a}$ is some ample vector bundle and $E_{f}$ is some finite vector bundle over $X$ (any of $E_{a}$ and $E_{f}$ are allowed to be zero).

Proof. We first consider the case where the characteristic of $k$ is zero.

Assume that $E=E_{a} \oplus E_{f}$, where $E_{a}$ is an ample vector bundle and $E_{f}$ a finite vector bundle over $X$. Since $E_{a}$ is ample, there is a nonconstant morphism of smooth projective curves

$$
f_{1}: Y_{1} \longrightarrow X
$$

such that $f_{1}^{*} E_{a}$ is globally generated (see Theorem 3.6). Since $E_{f}$ is a finite vector bundle, there is a finite étale Galois covering

$$
f_{0}: Y_{0} \longrightarrow X
$$

such that the vector bundle $f_{0}^{*} E_{f}$ is trivializable. Let $Y$ be the normalization of some connected component of the fiber product $Y_{1} \times_{X} Y_{0}$ over $X$. Let $f$ denote the natural projection of $Y$ to $X$. Since both $f^{*} E_{a}$ and $f^{*} E_{f}$ are globally generated, we conclude that the vector bundle $E=E_{a} \oplus E_{f}$ is virtually globally generated.

To prove the converse, let $E$ be a virtually globally generated vector bundle over $X$. Let $C$ be an irreducible smooth projective curve and

$$
\rho: C \longrightarrow X
$$

a nonconstant morphism such that $\rho^{*} E$ is globally generated.

Let

$$
0=E_{0}^{1} \subset E_{1}^{1} \subset \cdots \subset E_{\ell-1}^{1} \subset E_{\ell}^{1}=E
$$

be the Harder-Narasimhan filtration of the vector bundle $E$. Since $\rho$ in (4.3) is a finite morphism, the pull back to $C$ of a semistable vector bundle over $X$ remains semistable. Consequently, the pull back to $C$ of the filtration in (4.4) has the property that each successive quotient is semistable with decreasing slopes. Therefore, the filtration in (4.4) pulls back to the Harder-Narasimhan filtration of $\rho^{*} E$. Set

$$
E_{i}=\rho^{*} E_{i}^{1}
$$

for all $i \in[0, \ell]$. Therefore,

$$
0=E_{0} \subset E_{1} \subset \cdots \subset E_{\ell-1} \subset E_{\ell}=\rho^{*} E
$$

is the Harder-Narasimhan filtration of the vector bundle $\rho^{*} E$. 
Since $\rho^{*} E$ is globally generated, the quotient $\left(\rho^{*} E\right) / E_{\ell-1}$ is also globally generated. Hence

$$
\mu_{\min }\left(\rho^{*} E\right):=\mu\left(\left(\rho^{*} E\right) / E_{\ell-1}\right) \geq 0 .
$$

First assume that $\mu_{\min }\left(\rho^{*} E\right)>0$. Then $\rho^{*} E$ is ample (see Lemma 2.1(g)). From this it follows that $E$ is ample. Hence (4.1) holds with $E_{t}=0$.

Next consider the other case, namely $\mu_{\min }\left(\rho^{*} E\right)=0$, where $\rho$ is the morphism in (4.3). Let $r_{0}$ be the rank of $\left(\rho^{*} E\right)_{\min }:=\left(\rho^{*} E\right) / E_{\ell-1}$ (see (4.6)). Let

$$
q: \rho^{*} E \longrightarrow\left(\rho^{*} E\right) / E_{\ell-1}
$$

be the quotient homomorphism.

Fix a $k$-rational point $z_{0} \in C$, and also fix sections

$$
\beta_{j} \in H^{0}\left(C, \rho^{*} E\right),
$$

$j \in\left[1, r_{0}\right]$, such that $\left\{q\left(\beta_{1}\left(z_{0}\right)\right), \cdots, q\left(\beta_{r_{0}}\left(z_{0}\right)\right)\right\}$ span the fiber $\left(\left(\rho^{*} E\right)_{\min }\right)_{z_{0}}$ (recall that $\rho^{*} E$ is globally generated and $\left.r_{0}=\operatorname{rank}\left(\left(\rho^{*} E\right)_{\min }\right)\right)$. Consider the homomorphism

$$
\phi: \mathcal{O}_{C}^{\oplus r_{0}} \longrightarrow\left(\rho^{*} E\right)_{\min }
$$

defined by

$$
\phi\left(y ; \lambda_{1}, \cdots, \lambda_{r_{0}}\right)=\sum_{i=1}^{r_{0}} \lambda_{i} \cdot q\left(\beta_{i}(y)\right),
$$

where $y \in C, \lambda_{i} \in k$, and $q$ is defined in (4.7). This homomorphism $\phi$ induces a homomorphism of determinant line bundles

$$
\bigwedge^{r_{0}} \phi: \bigwedge^{r_{0}} \mathcal{O}_{C}^{\oplus r_{0}}=\mathcal{O}_{C} \longrightarrow \bigwedge^{r_{0}}\left(\rho^{*} E\right)_{\min } .
$$

Since degree $\left(\bigwedge^{r_{0}}\left(\rho^{*} E\right)_{\min }\right)=0$ and $\bigwedge^{r_{0}} \phi\left(z_{0}\right)$ is an isomorphism, we conclude that $\bigwedge^{r_{0}} \phi$ is an isomorphism of line bundles. Hence the homomorphism $\phi$ in (4.8) is an isomorphism.

Let

$$
\widehat{\phi}: \mathcal{O}_{C}^{\oplus r_{0}} \longrightarrow \rho^{*} E
$$

be the homomorphism defined by

$$
\widehat{\phi}\left(y ; \lambda_{1}, \cdots, \lambda_{r_{0}}\right)=\sum_{i=1}^{r_{0}} \lambda_{i} \cdot \beta_{i}(y),
$$

where $y \in C$ and $\lambda_{i} \in k$. Hence we have $q \circ \widehat{\phi}=\phi$. 
Since the homomorphism $\phi$ is an isomorphism, we conclude that the homomorphism $\widehat{\phi}$ gives a splitting

$$
\rho^{*} E=E_{\ell-1} \oplus \operatorname{image}(\widehat{\phi})=E_{\ell-1} \oplus\left(\rho^{*} E\right)_{\min }=E_{\ell-1} \oplus \mathcal{O}_{C}^{\oplus r_{0}} .
$$

For the filtration in (4.4), from (4.5) we have

$$
\mu_{\min }\left(E_{\ell-1}^{1}\right)=\mu_{\min }\left(E_{\ell-1}\right) / \operatorname{degree}(\rho)=\mu\left(E_{\ell-1} / E_{\ell-2}\right) / \operatorname{degree}(\rho)>0
$$

if $E_{\ell-1} / E_{\ell-2}$ in (4.6) is nonzero. Therefore, the vector bundle $E_{\ell-1}^{1}$ over $X$ is ample if it is nonzero (see Lemma 2.1(g)). Using the decomposition in (4.9) we will show that $E$ decomposes as a direct sum of $E_{\ell-1}^{1}$ and a finite vector bundle.

Consider the short exact sequence of vector bundles over $X$

$$
0 \longrightarrow E_{\ell-1}^{1} \longrightarrow E \longrightarrow E / E_{\ell-1}^{1} \longrightarrow 0
$$

We will prove that it splits. For that, take a pair $(\widetilde{C}, \tau)$, where $\widetilde{C}$ is a connected smooth projective curve defined over $k$ and

$$
\tau: \widetilde{C} \longrightarrow C
$$

a surjective map, such that the composition

$$
\widetilde{\rho}:=\rho \circ \tau: \widetilde{C} \longrightarrow X
$$

is a Galois covering. From the splitting in (4.9) we know that the pull back of the exact sequence in (4.10) to $C$ splits. Therefore, the pull back of the exact sequence in (4.10) to $\widetilde{C}$ also splits.

Fix a splitting

$$
\theta: \widetilde{\rho}^{*}\left(E / E_{\ell-1}^{1}\right) \longrightarrow \widetilde{\rho}^{*} E
$$

of the pulled back short exact sequence

$$
0 \longrightarrow \widetilde{\rho}^{*} E_{\ell-1}^{1} \longrightarrow \widetilde{\rho}^{*} E \longrightarrow \widetilde{\rho}^{*}\left(E / E_{\ell-1}^{1}\right) \longrightarrow 0
$$

over $\widetilde{C}$. The Galois group $\operatorname{Gal}(\widetilde{\rho})$ for the covering map in (4.11) has a natural action on any vector bundle over $\widetilde{C}$ which is pulled back from $X$. Furthermore, the exact sequence in (4.13), being a pull back of an exact sequence on $X$, is compatible with the actions of $\operatorname{Gal}(\widetilde{\rho})$ on the vector bundles in the exact sequence. In other words, all the homomorphisms in the exact sequence commute with the actions of the Galois group.

Therefore, for any element $\alpha \in \operatorname{Gal}(\widetilde{\rho})$, the pull back

$$
\alpha^{*} \theta: \widetilde{\rho}^{*}\left(E / E_{\ell-1}^{1}\right)=\alpha^{*} \widetilde{\rho}^{*}\left(E / E_{\ell-1}^{1}\right) \longrightarrow \alpha^{*} \widetilde{\rho}^{*} E=\widetilde{\rho}^{*} E
$$


is also a splitting of the short exact sequence in (4.13), where $\theta$ is as in (4.12). Consider the average homomorphism

$$
\widehat{\theta}:=\frac{1}{\# \operatorname{Gal}(\widetilde{\rho})} \sum_{\alpha \in \operatorname{Gal}(\widetilde{\rho})} \alpha^{*} \theta
$$

which is a splitting of the short exact sequence in (4.13). This homomorphism $\widehat{\theta}$ clearly commutes with the actions of $\operatorname{Gal}(\widetilde{\rho})$ on $\tilde{\rho}^{*}\left(E / E_{\ell-1}^{1}\right)$ and $\tilde{\rho}^{*} E$. From this it follows immediately that $\widehat{\theta}$ descends to a splitting of the short exact sequence in (4.10). In other words, we have

$$
E \cong E_{\ell-1}^{1} \oplus\left(E / E_{\ell-1}^{1}\right) .
$$

We have already shown that the vector bundle $E_{\ell-1}^{1}$ is ample (provided it is nonzero). We will now show that $E / E_{\ell-1}^{1}$ is a finite vector bundle. For that we first note that the pull back of $E / E_{\ell-1}^{1}$ to $C$ is trivializable (recall that the homomorphism $\phi$ in (4.8) is an isomorphism). Therefore, the vector bundle $\widetilde{\rho}^{*}\left(E / E_{\ell-1}^{1}\right)$ over $\widetilde{C}$, which coincides with $\tau^{*}\left(\rho^{*} E\right)_{\min }$, is trivializable. To prove that the vector bundle $E / E_{\ell-1}^{1}$ is finite, we need to produce an étale Galois covering of $X$ such that the pull back of $E / E_{\ell-1}^{1}$ to it is trivializable. Although the covering $\widetilde{\rho}$ may not be étale, we will construct a quotient of $\widetilde{C}$ which is an étale Galois covering of $X$.

We will define an equivalence relation on the $k$-rational points of $\widetilde{C}$ in the following way. Two points $y_{1}$ and $y_{2}$ are equivalent if the following two conditions hold:

- $\widetilde{\rho}\left(y_{1}\right)=\widetilde{\rho}\left(y_{2}\right)$, and

- the identification of the fiber $\left(\widetilde{\rho}^{*}\left(E / E_{\ell-1}^{1}\right)\right)_{y_{1}}$ with $\left(\widetilde{\rho}^{*}\left(E / E_{\ell-1}^{1}\right)\right)_{y_{2}}$ given by any trivialization of the vector bundle $\widetilde{\rho}^{*}\left(E / E_{\ell-1}^{1}\right)$ coincides with the identity map of $\left(E / E_{\ell-1}^{1}\right) \widetilde{\rho}\left(y_{1}\right)$. It is easy to see that this condition does not depend on the choice of the trivialization of $\widetilde{\rho}^{*}\left(E / E_{\ell-1}^{1}\right)$. (As both $\left(\widetilde{\rho}^{*}\left(E / E_{\ell-1}^{1}\right)\right)_{y_{1}}$ and $\left(\widetilde{\rho}^{*}\left(E / E_{\ell-1}^{1}\right)\right)_{y_{2}}$ are identified with $\left(E / E_{\ell-1}^{1}\right) \tilde{\rho}\left(y_{1}\right)$, an isomorphism of $\left(\widetilde{\rho}^{*}\left(E / E_{\ell-1}^{1}\right)\right)_{y_{1}}$ with $\left(\widetilde{\rho}^{*}\left(E / E_{\ell-1}^{1}\right)\right)_{y_{2}}$ gives an automorphism of $\left(\widetilde{\rho}^{*}\left(E / E_{\ell-1}^{1}\right)\right)_{y_{1}}$.)

The quotient of $\widetilde{C}$ by this equivalence relation gives an étale Galois covering of $X$ with the property that the pull back of $E / E_{\ell-1}^{1}$ to it is trivializable.

Therefore, the vector bundle $E / E_{\ell-1}^{1}$ over $X$ is finite. Hence, the decomposition of $E$ in (4.14) is a decomposition of the type as in (4.1). In other words, (4.1) holds. This completes the proof of the theorem under the assumption that the characteristic of $k$ is zero.

We now assume the characteristic of $k$ to be positive.

Let $E$ be a vector bundle over $X$ and $m$ an integer such that $\left(F_{X}^{m}\right)^{*} E \cong$ $E_{a} \oplus E_{f}$ as in (4.2). Since $E_{a}$ is ample, we know that $E_{a}$ is virtually globally 
generated [BP, Theorem 2.2]. Since $E_{f}$ is finite, it is also virtually globally generated. Therefore, $\left(F_{X}^{m}\right)^{*} E \cong E_{a} \oplus E_{f}$ is virtually globally generated. Since $\left(F_{X}^{m}\right)^{*} E$ is virtually globally generated, we conclude that $E$ itself is virtually globally generated.

To prove the converse, let $E$ be a virtually globally generated vector bundle over $X$. There is an integer $m$ such that

$$
\left(F_{X}^{m}\right)^{*} E=\bigoplus_{i=1}^{\ell} V_{i}
$$

where each $V_{i}$ is a strongly semistable vector bundle over $X$ [BP, Proposition 2.1].

The vector bundle $\left(F_{X}^{m}\right)^{*} E$ is virtually globally generated as $E$ is so. Hence each $V_{i}$ in (4.15) is virtually globally generated. Therefore,

$$
\operatorname{degree}\left(V_{i}\right) \geq 0
$$

for all $i \in[1, \ell]$.

If degree $\left(V_{i}\right)>0$, then the strongly semistable vector bundle $V_{i}$ is ample [Bi2, Theorem 1.1] (similar results were proved in [La], [Ba], [Br], [Lan]).

Assume that degree $\left(V_{i}\right)=0$. Since $V_{i}$ is virtually globally generated, there is a smooth projective curve $Y$ and a finite morphism

$$
f: Y \longrightarrow X
$$

such that $f^{*} V_{i}$ is globally generated. Since

$$
\operatorname{degree}\left(f^{*} V_{i}\right)=\operatorname{degree}(f) \cdot \operatorname{degree}\left(V_{i}\right)=0,
$$

the vector bundle $f^{*} V_{i}$ is trivializable. This is proved by repeating the earlier argument showing that the homomorphism $\phi$ in (4.8) is an isomorphism.

Since $f^{*} V_{i}$ is trivializable, it follows that the vector bundle $V_{i}$ is essentially finite.

Set $\delta_{i}=\operatorname{degree}\left(V_{i}\right)$. Consider the decomposition

$$
\left(F_{X}^{m}\right)^{*} E=\bigoplus_{i=1}^{\ell} V_{i}=\left(\bigoplus_{\delta_{i}>0} V_{i}\right) \oplus\left(\bigoplus_{\delta_{i}=0} V_{i}\right)=: W_{a} \oplus W_{e}
$$

obtained using (4.15) and (4.16). We have shown above that $W_{a}$ is ample and $W_{e}$ is essentially finite. Since $W_{e}$ is essentially finite, for all integers $n$ sufficiently large, the pull back $\left(F_{X}^{n}\right)^{*} W_{e}$ is finite vector bundle. Consider

$$
\left(F_{X}^{m+n}\right)^{*} E=\left(F_{X}^{n}\right)^{*} W_{a} \oplus\left(F_{X}^{n}\right)^{*} W_{e},
$$

where $n$ is sufficiently large. Since $F_{X}$ is a finite morphism and $W_{a}$ is ample, the vector bundle $\left(F_{X}^{n}\right)^{*} W_{a}$ is ample. In other words, (4.17) is a decomposition of type (4.2). Thus, any virtually globally generated vector bundle admits a decomposition of type (4.2). This completes the proof of the theorem. 
Remark 4.2. Fix distinct $k$-rational points $D:=\left\{p_{1}, \cdots, p_{d}\right\}$ of a smooth projective curve $X$. A parabolic vector bundle over $X$ with parabolic structure over $D$ is a vector bundle $E$ over $X$ together with a decreasing filtration of subspaces

$$
V_{1}^{i} \subset V_{2}^{i} \subset \cdots \subset V_{\ell_{i}}^{i}=E_{p_{i}}
$$

of each fiber $E_{p_{i}}$, and furthermore, to each subspace $V_{j}^{i}$ there is an associated rational number $0 \leq \lambda_{j}^{i}<1$ such that $\lambda_{j}^{i}>\lambda_{j+1}^{i}$ for all $i, j$; see $[\mathrm{MS}]$ for the details. The notion of an ample vector bundle can be extended to the context of parabolic bundles (see [Bi1]). Parabolic vector bundles correspond to orbifold vector bundles, i.e., equivariant vector bundles equipped with an action of a finite group. For an ample parabolic bundle, the corresponding orbifold vector bundle is ample in the usual sense [Bi1, Lemma 4.6]. Using this it can be shown that Theorem 4.1 generalizes to the context of parabolic bundles.

\section{References}

[Ba] C. M. BARTON, Tensor products of ample vector bundles in characteristic p, Amer. J. Math. 93 (1971), 429-438.

[Bi1] I. BISWAS, Parabolic ample bundles, Math. Ann. 307 (1997), 511-529.

[Bi2] I. BISWAS, A criterion for ample vector bundles over a curve in positive characteristic, Bull. Sci. Math. 129 (2005), 539-543.

[BP] I. BISWAS and A. J. PARAMESWARAN, On the ample vector bundles over curves in positive characteristic, C.R. Acad. Sci. Paris Sér. I Math. 339 (2004), 355-358.

[Br] H. BRENNER, Slopes of vector bundles on projective curves and applications to tight closure problems, Trans. Amer. Math. Soc. 356 (2004), 371-392.

[Ha1] R. HARTShorne, "Ample Subvarieties of Algebraic Varieties", Lecture Notes in Mathematics, Vol. 156, Springer-Verlag, Berlin-New York, 1970.

[Ha2] R. HARTSHORne, Ample vector bundles on curves, Nagoya Math. J. 43 (1971), 73-89.

[La] H. LANGE, On stable and ample vector bundles of rank 2 on curves, Math. Ann. 238 (1978), 193-202.

[Lan] A. LANGER, Semistable sheaves in positive characteristic, Ann. of Math. (2) 159 (2004), 251-276.

[MS] V. B. Mehta and C. S. SEShADRI, Moduli of vector bundles on curves with parabolic structures, Math. Ann. 248 (1980), 205-239.

[Mu] D. Mumford, Pathologies III, Amer. J. Math. 89 (1967), 94-104.

[No] M. V. NORI, On the representations of the fundamental group scheme, Compositio Math. 33 (1976), 29-41.

[Pa] A. J. PARAMESWARAN, Virtual global generation of ample bundles over curves, unpublished manuscript.

[RR] S. Ramanan and A. Ramanathan, Some remarks on the instability flag, Tôhoku Math. J. (2) 36 (1984), 269-291.

School of Mathematics

Tata Institute of Fundamental Research

Homi Bhabha Road

Mumbai 400005, India

indranil@math.tifr.res.in

param@math.tifr.res.in 